\title{
DISEÑO DE UN DISPOSITIVO ELECTRÓNICO DE ACCIONES CÍCLICAS COMO HERRAMIENTA DE ENTRETENIMIENTO PARA LA INCLUSIÓN SOCIAL DE PERSONAS EN ESTADO DE CUADRIPLEJÍA
}

\author{
DESIGN OF AN ELECTRONIC DEVICE BASED ON CYCLIC ACTIONS AS AN \\ ENTERTAINMENT TOOL FOR SOCIAL INCLUSION TO PEOPLE ON QUADRIPLEGIA \\ CONDITION
}

\section{Resumen}

La inclusión social se presenta como factor fundamental dentro del desarrollo social; las personas con algún tipo de discapacidad son excluidas de un entorno colectivo. En Colombia se encuentran alrededor de 260.000 casos de cuadriplejía, discapacidad física ocasionada por parálisis del tronco, piernas y brazos. Por lo anterior, se plantea en este trabajo el diseño de un dispositivo orientado al desarrollo físico y emocional que se genera a partir del entretenimiento, permitiéndole al usuario en desventaja la integración con las personas de su núcleo familiar, a través de un videojuego. BUCLE es un dispositivo de control, que identifica los movimientos de la columna cervical como comandos de interacción en un videojuego. Este dispositivo se evaluó con 10 personas, una de ellas en estado de cuadriplejía. Se observó que el usuario, al interactuar con la interface, mantiene la visualización sin ningún problema; además se observa una diferencia de solo un $5 \%$ de la eficacia con respecto a comandos realizados por personas sin limitaciones físicas.

Palabras clave: Discapacidad, Videojuego, inclusión social, Columna Cervical, cuadriplejía.

Pablo A. Gelvez $\mathbf{M}^{1}$ Harold M. Torres C2 Juan C. Moreno $\mathbf{M}^{3}$ Luis E. Bautista $\mathbf{R}^{4}$

Recibido: 15 de septiembre de 2013 Aceptado: 20 de diciembre de 2013

\section{Abstract}

Social inclusion is presented as a fundamental factor a social development. People with disabilities are excluded from collective environments. There are around 260,000 cuadriplejía cases in Colombia, a physical disability caused by paralysis in trunk, legs and arms. This paper presents a design of a device oriented to physical and emotional development which is generated thanks to entertainment, allowing disable people to integrate with their nuclear family via a video game. BUCLE is a control device, which identifies the cervical spine movements as interaction commands in a video game. This device was evaluated by 10 people, one of them on quadriplegia condition. It was observed that the user interacting with the interface maintains the display without any problem; also a difference of only $5 \%$ efficiency with respect to commands made by people without physical limitations are observed.

Key words: Disability, Video Game, social inclusion, Cervical Spine, quadriplegia condition.

\footnotetext{
1 E-mail: pablog14@gmail.com

2E-mail:khandos@gmail.com

3E-mail:fedor@uis.edu.co

4 E-mail: Iuis.bautista@correo.uis.edu.co

1,2Estudiantes de Diseño Industrial 3,4 Docentes Investigadores, Grupo de Investigación INTERFAZ. Universidad Industrial de Santander
} 


\section{Introducción}

La inclusión social de personas con discapacidad es un factor que, dentro de los contextos de sociedad, llámense grupos familiares, grupos de amistades o relaciones de trabajo, resulta fundamental, dado que la mayoría de casos termina por excluir de su entorno a dichas personas, limitándolas a ser simples testigos de actividades que podrían desarrollar con ayudas tecnológicas.

En Colombia se ven afectadas en el movimiento del cuerpo, manos, brazos y piernas cerca de 260.000 personas (DANE, 2010). Dichas afecciones también conocidas como cuadriplejía, que se refieren a la parálisis del cuello hacia abajo, incluyendo el tronco, las piernas y los brazos (Spine-health, 2013).

De otro lado, se ha demostrado que los videojuegos poseen cualidades sicológicas favorables ante diversas situaciones de estrés y afectación emocional; además tienen efectos positivos como mejorar el equilibrio, favorecer la organización espacio-temporal, mejorar la coordinación, promover el espíritu de superación, estimular la concentración, y fortalecer el trabajo en equipo. Lo anterior supone un paso para generar inclusión social, donde se espera que las personas puedan interactuar, sin que tenga necesariamente relación con la rehabilitación e independientemente de las diferencias en sus capacidades motoras, sensoriales, cognitivas o psíquicas (D Rand, 2012).

En la actualidad existen diversas propuestas: una de ellas busca interpretar el movimiento ocular y convertirlo en señales electrónicas (The eye writer, 2014; Veckers, 2012), otro modelo se enfoca en el uso de la boca para realizar mecánicas convertibles en señales digitales (Yankelevitz, 2010). Zhendou (2000), plantea un sistema de control bajo un único mando donde se concentran todas las acciones para controlar dispositivos electrónicos alrededor del usuario. Existen otras propuestas mas completas como la de un dispositivo acoplable a un control comercial de consola, basado en sensores que rastrean hasta 9 movimientos del jugador (Fanucci \& Roncella, 2011).

Algunos dispositivos para personas en condiciones de discapacidad similares son los propuestos por Zavala \& Favela (2012), quienes desarrollaron un dispositivo para que adultos mayores con movilidad reducida interactúen con un videojuego, mediante un dinamómetro clínico; mientras que, aplicados a la domótica, Plaza \& Aperador (2013) desarrollaron un dispositivo usando técnicas de visión por computador y análisis de imágenes infrarrojas, aplicado en el control del entorno inmediato. Algunos autores han abordado esta condición desde el diseño de aplicaciones software con características especiales (Grammenos \& Savidis, 2009), proponiendo un enfoque de diseño de videojuegos acccesibles.

En este trabajo se presenta un dispositivo electrónico de acciones cíclicas Ilamado BUCLE, que toma su nombre de acuerdo a dichas acciones y el cual propone la inclusión social de personas en estado de cuadriplejía, mediante una herramienta que le permite a este sector de la población interactuar con su entorno familiar; además brinda acceso al campo de la lúdica a personas que conservan movimientos a nivel de columna cervical, aplicando para ello la tecnología.

En éste caso se consideró a una persona con lesión medular en las vértebras cervicales $C 4$ y/o C5, pues si no se presenta fractura cervical, estas personas a menudo conservan el control de hombros y bíceps, y aunque no controlan la mano o el puño, no necesitan sistemas auxiliares permanentes para la respiración. Además sus funciones musculares en cuello y pectorales, según el trauma y rehabilitación, son muy cercanas al promedio de una persona sin lesión. 
Durante el proceso de diseño se tuvieron en cuenta las condiciones de una persona como la descrita anteriormente, de modo que se lograron establecer las limitaciones de movimiento. Posteriormente se realizó un desarrollo técnico mediante un dispositivo electrónico que, usando sensores de movimiento, le permitieron a un paciente con cuadriplejía controlar una interfaz de 6 funciones, activadas por el movimiento de la cabeza, para interactuar con el videojuego SIDE POCKET - Billar Pool (Nintendo, 2013). Por medio de un test, en Bucle se evaluaron la visualización y la eficacia. De esta manera se pudo confirmar que la persona logra realizar las funciones de interacción de control del videojuego, con una eficacia del 56\%. Así mismo, se evidenció que BUCLE puede reconocer un rango muy preciso de movimientos.

\section{Materiales y métodos}

\subsection{Cuadriplejía y rehabilitación}

La cuadriplejía es causada principalmente por lesiones en la médula espinal, pero también pueden ser causadas por enfermedades del sistema nervioso. La mayoría de las lesiones medulares se producen de repente, como consecuencia de un traumatismo $y$, aproximadamente, en la mitad de los casos el origen es un accidente de tráfico (Discapnet, 2013).

Como síntomas predominantes se evidencian: la invalidez, vejiga e intestino neurogénico con incontinencia urinaria y fecal, disfunción eréctil, falta de movilidad y de sensibilidad. Por estas limitaciones, las personas en condición de cuadriplejía necesitan ayuda de terceros para todas las actividades de su vida diaria, incluyendo silla de ruedas para sus desplazamientos; debido a lo anterior, se pueden presentar trastornos depresivos y psicóticos.

Las funciones motoras residuales para personas con lesión medular completa a nivel de las vértebras $C 4$ - $C 5$ y $C 6$, generalmente se limitan a la columna cervical, CC, y movimientos restringidos de la cintura escapular, como se ilustra en la figura 1.

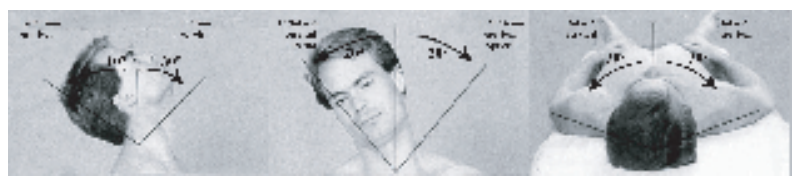

Figura 1. Rangos seguros para el Movimientos de la $\mathrm{CC}$ en personas con lesiones a nivel de C4 y C5 (Patiño, 2013; Maikel, 2012)

La rehabilitación de personas con discapacidad se puede abordar desde diferentes aspectos. Así, la intervención sicológica se centra en un único objetivo, el de mantener al paciente con un buen estado anímico, a través de diálogos que mantienen consciente a la persona de su recuperación o posible mejoría con el paso del tiempo. Si no se interviene anímicamente a los pacientes cuadripléjicos, estos pueden comenzar una etapa de deterioro de sus condiciones actuales, se puede presentar depresiones las cuales conllevan pérdidas de las condiciones físicas y mentales. El problema de la discapacidad no está limitado únicamente a la persona, involucra también a la familia, a la comunidad, a la sociedad y a la cultura en general (Céspedes, 2005).

Desde el punto de vista del trabajo social, el objetivo en los pacientes jóvenes es conseguir una función completa y sin limitaciones y, en los de más edad, recuperar la capacidad de realizar el mayor número de actividades cotidianas posibles. Se determinan los objetivos funcionales de cada paciente, según el nivel medular afectado. El potencial funcional también depende de otros factores como: la edad, enfermedades asociadas, medios técnicos existentes y la motivación del paciente. En los pacientes con discapacidad hay tendencia a la depresión y se puede perder la motivación para recuperar la función perdida.

En cuanto a la fisioterapia, antes de realizarla se debe evaluar el "arco de movilidad" de las regiones 
afectadas. Para ello, primero se realizan ejercicios de estiramiento mantenido para relajar los músculos que presentan contracturas. Los ejercicios de arco movilidad pueden ser: Activos cuando el paciente puede realizar el ejercicio sin ayuda-, Asistidos -cuando los músculos son muy débiles o cuando la movilidad articular produce dolor-, y Pasivos -cuando el paciente no puede participar activamente-.

\subsection{Estado actual de las interfaces}

\section{QUADCONTROL}

Actualmente Quadcontrol ofrece dos tipos de joysticks adaptados para SONY PlayStation 2 y Microsoft Xbox 360. El adaptado a la consola de Microsoft ofrece, por ejemplo, un botón para cambiar el joystick bucal entre el control "D" y otro para cambiar entre los controles análogos derecho e izquierdo, dos switches activados con los labios para $L 3$ y $R 3$, además de un tubo para sorber y soplar (sip/puff), tal como se observa en la figura 2.

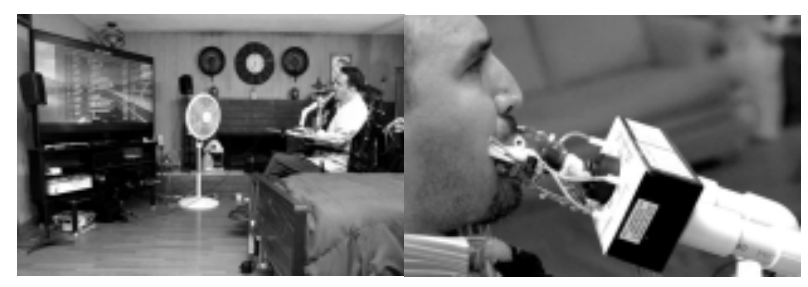

Figura 2. A) Entorno del paciente, b) Detalle de la interface QuadControl (Yankelevitz, 2010)

Aunque durante años se ha investigado la manera de utilizar los ojos para manejar aparatos de computación, se hace necesario que el sistema sea accesible y asequible para que las personas que no tienen movilidad, puedan 'presionar' botones solo mirando la pantalla y darles movimiento a personajes hacia diferentes sitios, ver figura 3.

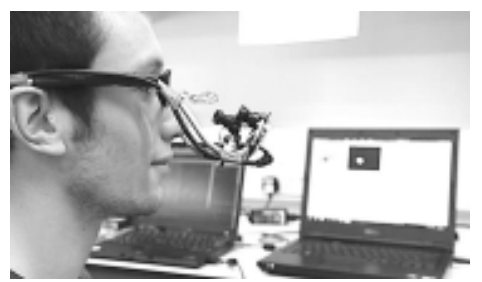

Figura 3. Videojuego Stephen Vickers (Veckers, 2012)

\section{The eye writer}

Los integrantes de Free Art and Technology (FAT), OpenFrameworks, The Graffiti Research Lab. Y las comunidades EbelingGroup han conformado un equipo de trabajo con un legendario creador de grafitis, publicista y activista llamado TEMPTONE. Él fue diagnosticado con ELA en 2003, una enfermedad que lo ha dejado casi completamente paralizado, excepto sus ojos.

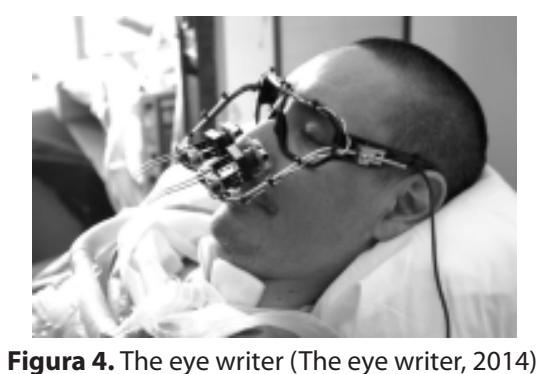

Este equipo está trabajando para crear, a bajo costo, el sistema de seguimiento ocular de código abierto que permitirá a los pacientes con ELA dibujar usando sólo sus ojos, ver figura 4.

\section{Metodología}

Para iniciar se analizó la posición que se debía asumir frente a la discapacidad física presente en los usuarios que se beneficiarán del trabajo realizado; se concluyó que tomar parte en la inclusión social es un punto clave del proyecto, puesto que la concienciación para el trabajo con discapacitados es pieza importante de la labor conjunta con este sector de la población. En la figura 5 se relacionan las etapas metodológicas seguidas en el desarrollo del proyecto.

Para tener acierto en el proceso de diseño, se llevaron a cabo tareas de manera concurrente, estableciendo un protocolo de trabajo para abordar cada uno de los pasos que se seguirían en el transcurso del proyecto: Indagación, establecimiento de requerimientos, alternativas de posibles soluciones, pruebas de carácter técnico a nivel de ingeniería electrónica y pruebas ergonómicas y de usabilidad. 


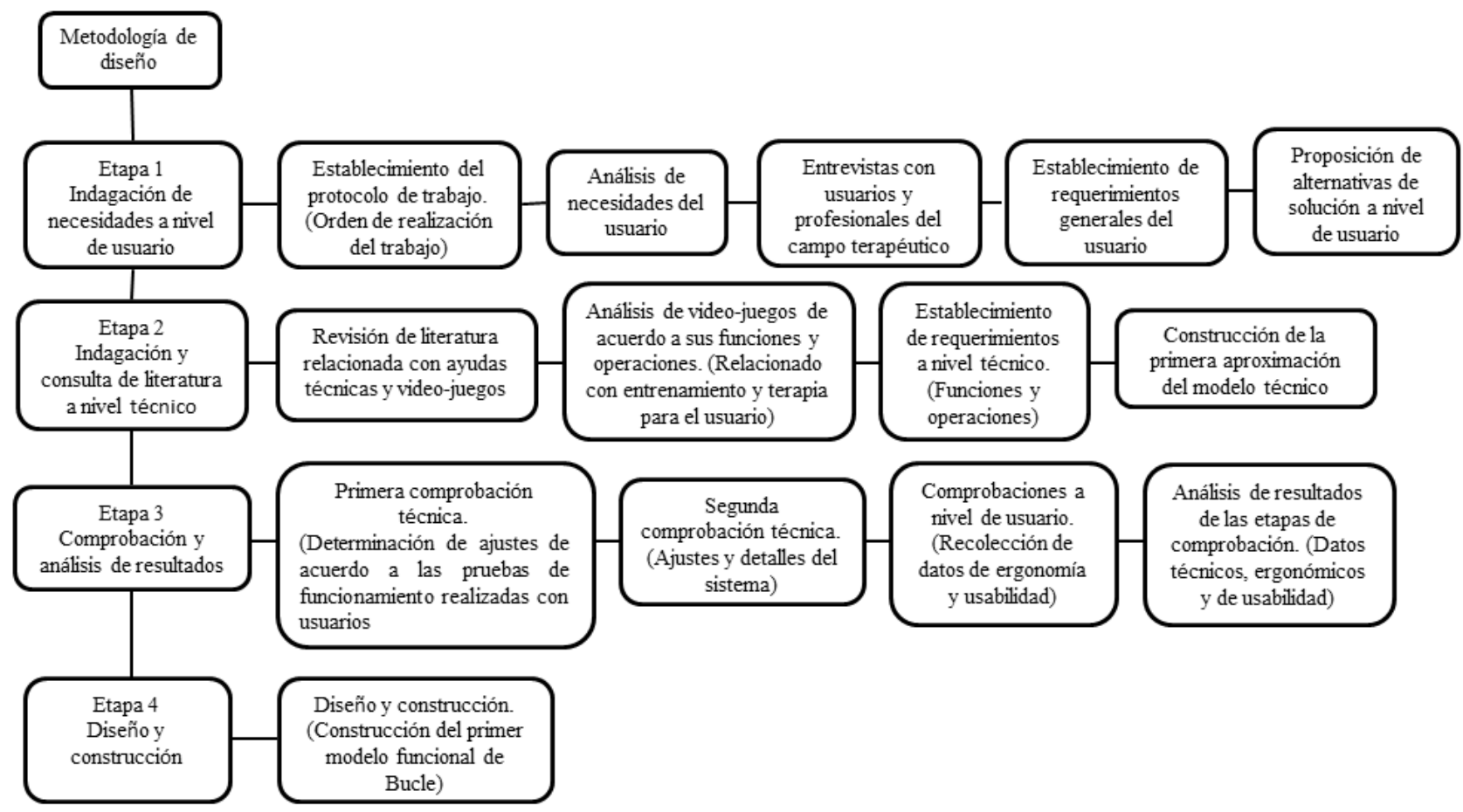

Figura 5. Metodología de Diseño (Huleves et al., 2009)

Aplicando el protocolo propuesto, se determinaron requerimientos y alternativas de diseño, establecidos a partir de la indagación, entrevistas con usuarios en estado de cuadriplejía, también con profesionales de la rama terapéutica y visitas de campo donde se recolectó información fundamental propia del entorno de la discapacidad de los usuarios, direccionando el proyecto para llegar a obtener resultados acordes a las necesidades establecidas.

Se abordó el proyecto mediante la revisión y análisis de literatura referente a las ayudas técnicas para personas en condición de vulnerabilidad y el diseño universal, además de literatura y material referente a los videojuegos y la interacción de estos con la población objetivo. Se analizaron los videojuegos desde el punto de vista de las funciones y operaciones necesarias para el desarrollo entretenido y terapéutico que implica la interacción con esta tecnología de una o más personas con o sin discapacidad.

Luego se establecieron los requerimientos de carácter técnico, a través de métodos del diseño concurrente e interdisciplinar con la ingeniería electrónica. Se establecieron Diagramas de Operaciones y Funciones, necesarios como medio de comunicación eficiente entre diseño e ingeniería, además de explicar tal como su nombre lo indica, qué operación deberá cumplir cierto mando luego de haberle asignado una función correspondiente. Estas etapas direccionaron la primera comprobación técnica, orientada a determinar la pertinencia del principio del funcionamiento electrónico.

Para la realización de la primera comprobación técnica, se hizo un primer acercamiento al usuario objetivo, familiarizándolo con el dispositivo en modo de prueba, y así poder capturar la información requerida para la disposición de mandos y señales necesarias para la interacción de control. El usuario generó las entradas para efectuar la medición a través de los movimientos de cuello y cabeza mostrados en la figura 1. En esta prueba, las señales de salida fueron numéricas y correspondían a un sistema electrónico de datos, 
por lo que dichos datos fueron objeto de posterior análisis por parte del grupo de desarrollo. Mediante el análisis gráfico de los umbrales de movimiento, llevado a cabo con el video y ayudas técnicas gráficas, se pudo determinar la relación entre la señal adquirida y el movimiento realizado por el paciente.

Posteriormente se diseñaron y aplicaron las pruebas ergonómicas, orientadas a validar las hipótesis de interacción planteadas por el equipo de diseño. Mediante dichas pruebas se determinó el nivel de comprensión del lenguaje de uso y con los resultados se pudo reorientar el diseño de manera adecuada.

\subsection{Interface propuesta}

Aprovechando la capacidad de movimiento de la columna cervical a nivel de C4 y C5 (operaciones) y los tipos de señales adquiridas por el componente electrónico, se planteó un principio de funcionamiento que aprovechara dichos movimientos como mecanismo de control de acciones. Teniendo en cuenta las acciones de control requeridas por el videojuego (funciones), se correlacionaron los movimientos de la columna cervical con dichas acciones de control, evaluándolas una a una, de manera que el usuario las reconociera e interpretara de manera natural. En la tabla 1 y figura 6 , se muestran las correlaciones entre operaciones (movimientos) y funciones (acciones) propuestas en El desarrollo del dispositivo periférico para el videojuego, se basó en tres requerimientos técnicos esenciales: el número de funciones establecidas para la

\begin{tabular}{cc}
\hline OPERACIONES & FUNCIONES \\
\hline Extensión Cervical/(Mentón Arriba) & Amba \\
Flexión Cervical/(Mentón Abajo) & Abajo \\
Inclinación Cervical hacia la & tzquierda \\
Inclinación Cervical hacia la derecha & \\
Rotación Cervical hacia la derecha & Derecha \\
Rotación Cervical hacia la izquierda & Empezar/Pausa(START/PAUSE)
\end{tabular}

Tabla 1. Correlación entre Operaciones y Funciones

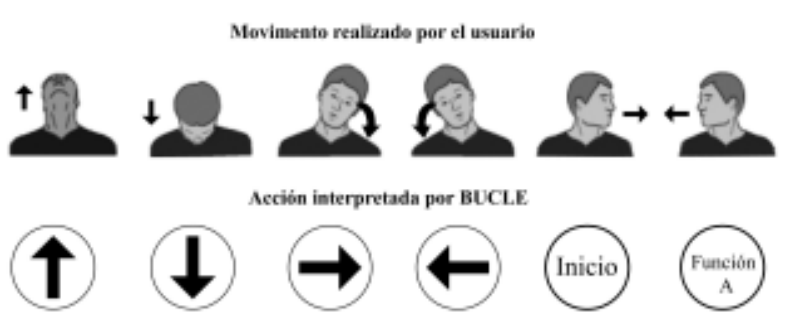

Figura 6. Funciones de Movimiento

interacción, la reacción de interacción del usuario sin límite de tiempo y la retroalimentación adecuada de las acciones. Se efectuó la revisión de videojuegos para la consola Nintendo ${ }^{\circledR}$, que cumplieran con estos requerimientos. El primer requerimiento lo cumplen las 3 primeras generaciones de videojuegos, luego se filtraron teniendo en cuenta el segundo requerimiento, encontrando que 20 juegos lo cumplen. Finalmente, se filtró el último requerimiento y se encontró que el videojuego SIDE POCKET (Nintendo, 2013), cumplía con todas las exigencias. En la figura 7 se observa la interfaz gráfica del videojuego seleccionado.

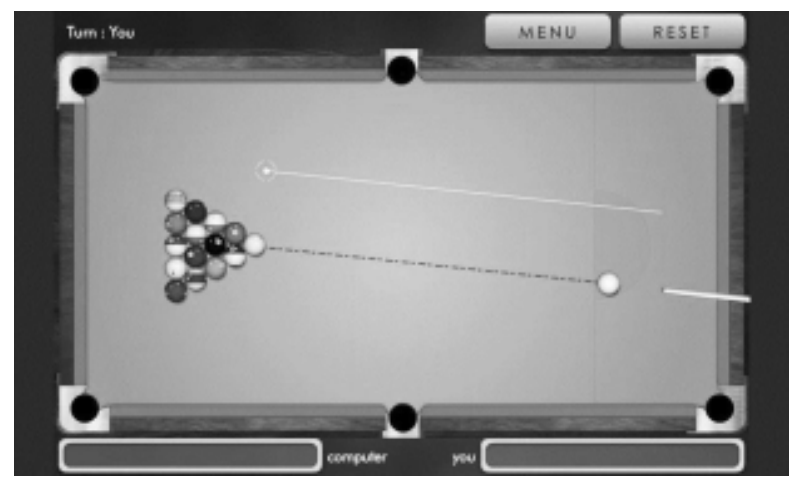

Figura 7. Visualización del Videojuego a ser usado con Bucle (Nintendo, 2013)

Partiendo del videojuego seleccionado, se ajustó el funcionamiento para trabajar con sensores de movimiento y microcontroladores. Las funcionalidades se basan en el uso de un acelerómetro para las funciones de dirección y un giroscopio para las dos funciones restantes, estos sensores están ubicados en la cabeza del usuario, sujetos por una diadema. Los sensores convierten en señales electrónicas codificadas el movimiento voluntario de la columna cervical. El dispositivo 


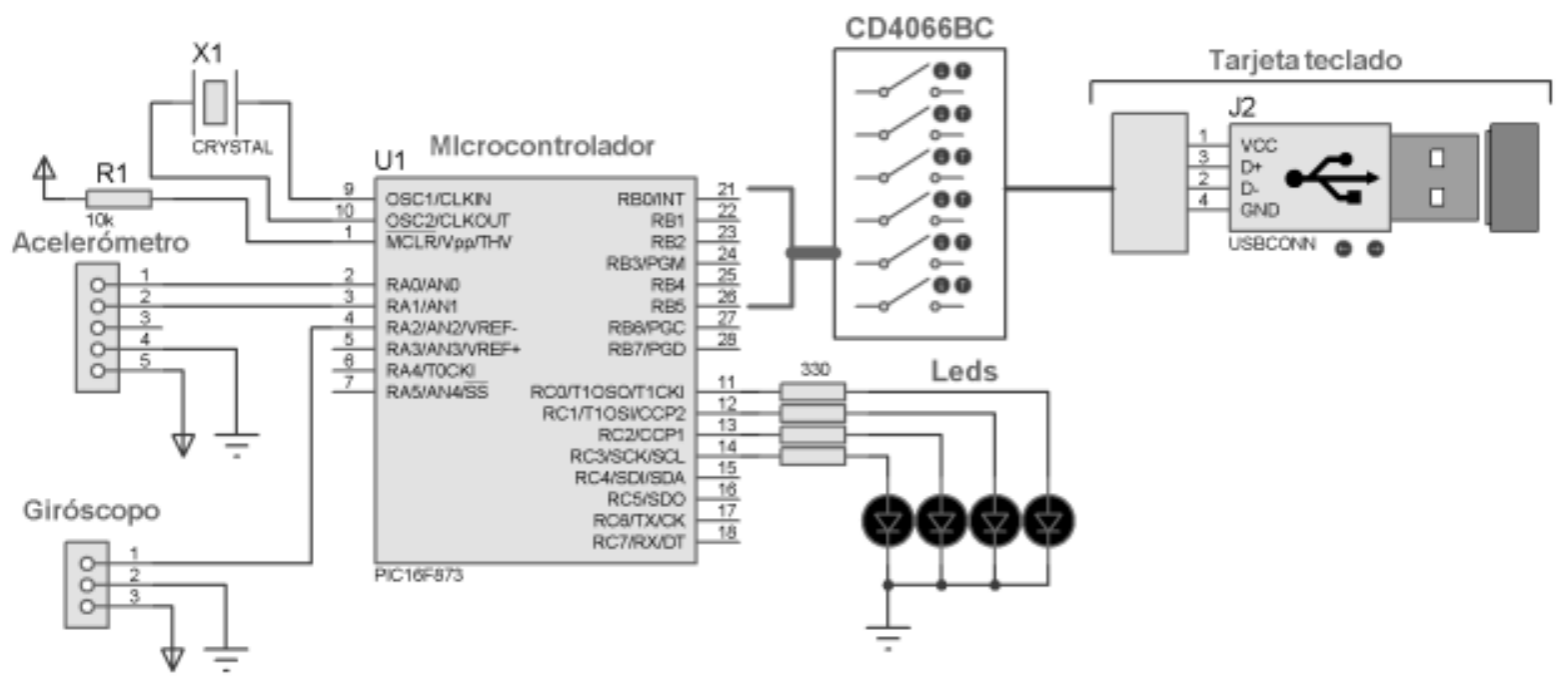

Figura 8. Diagrama electrónico de BUCLE

usa el puerto de datos USB para el envío y recepción de señales. En la figura 8, se observa el diagrama esquemático usado en BUCLE

\subsection{Población y pruebas}

Se realizaron dos pruebas con usuarios; para ello se desarrolló una aplicación informática que mostraba una serie de comandos en la interfaz gráfica, los cuales debían ser seguidos por el usuario. Este material consiste en una aplicación informática que estimula al usuario ante la tarea a ejecutar. La Prueba 1, P1, se realizó usando el teclado como periférico de entrada, empleando las teclas de flechas, Tecla A (función A) y Tecla Enter (start). En la Prueba 2, P2, se utilizó el dispositivo propuesto con las acciones relacionadas en la tabla 1.

Las pruebas se llevaron a cabo con 10 personas ( 4 hombres y 6 mujeres) en condiciones físicas y mentales suficientes para las tareas requeridas. La prueba 2 se realizó con una persona en condición de cuadriplejía con movimiento en columna cervical (al que se llamará Johnatan). Todos ciudadanos colombianos con edades entre 19 y 26 años.

El protocolo de la prueba fue el siguiente: $P 1$ inició con la lectura, por parte del usuario, del consentimiento informado donde se le explica el objetivo de la prueba y la forma en que se utilizará la información recolectada. Luego se explicó la tarea que desarrollaría, seguidamente se habilitó la aplicación informática mostrada en la figura 9. A continuación se le solicitó al usuario desarrollar la tarea, que consistía en seguir la secuencia de imágenes mostradas en la pantalla, utilizando el teclado del computador, tal como se observa en la figura 10.

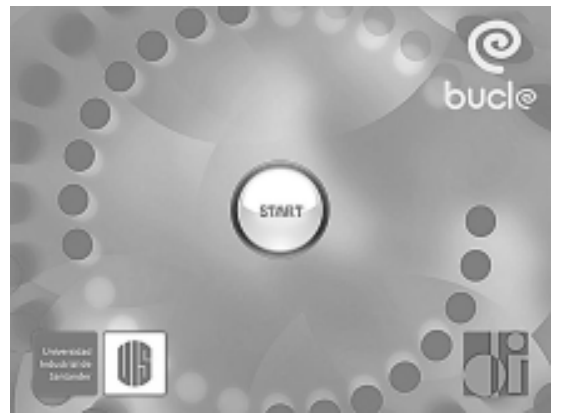

Figura 9. Aplicación diseñada para la prueba 


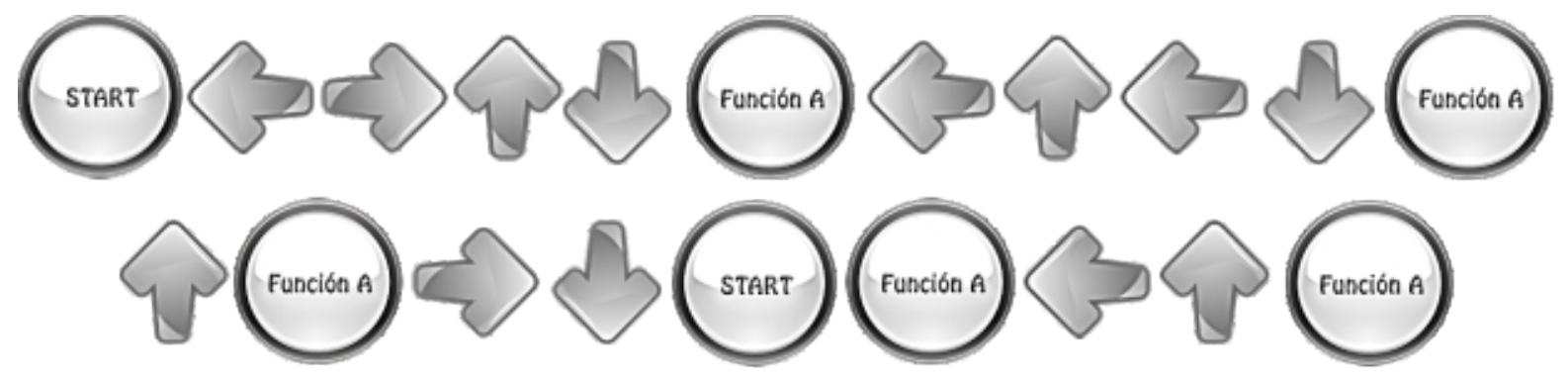

Figura 10. Secuencia de imágenes de la prueba

Para $P 2$ se repitió el mismo protocolo; sin embargo, como periférico de entrada se utilizó el dispositivo propuesto con el cual el usuario siguió los comandos mediante los movimientos del cuelloy la cabeza.

\section{Resultados y discusión}

\subsection{Dispositivo BUCLE}

El primer modelo funcional de BUCLE se obtuvo luego de establecer las alternativas resultantes a partir de los requerimientos y parámetros de diseño. Se evaluaron tres alternativas, a partir de un análisis de Despliegue de la Función Calidad, QFD, donde los datos resultantes dieron como mejor opción el dispositivo mostrado en la figura 11.

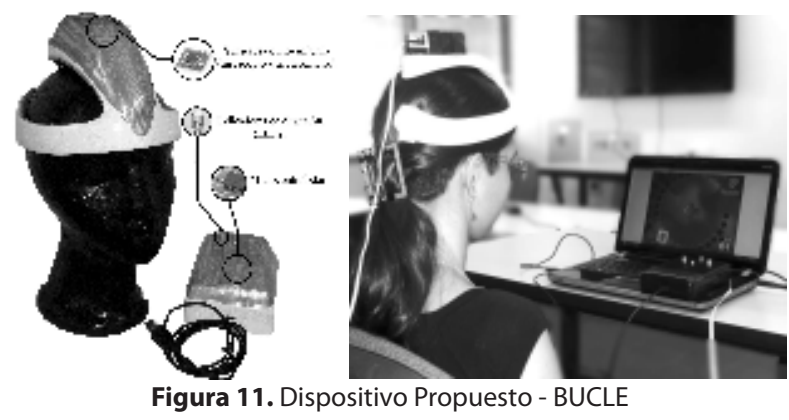

\subsection{Cuantificación de la Eficacia}

La eficacia cuantificada se determinó a partir de la capacidad del usuario para completar la tarea satisfactoriamente a través de la ecuación 1. El tiempo que el usuario gasta en completar la tarea, al igual que la cantidad de aciertos y errores, fueron capturados por la aplicación, y a partir de esos valores se calculó la Finalización satisfactoria con la ecuación 2.

Eficacia $=[$ Tiempo de la Prueba $(T P) *$ Finalización Satisfactoria $(F S)] / 100$ Ec. (1)

Finalización Satisfactoria (FS) $=[(\#$ de Aciertos) $-(\#$ de Errores/2)]*10 Ec. (2)

\subsection{Resultados}

Las pruebas permitieron evaluar cuantitativamente el tiempo de respuesta en el desarrollo de la tarea, cuyos resultados se muestran en las figuras 12 a 14, donde el nombre de los usuarios fue cambiado para proteger su identidad. En la figura 12 se relacionan los resultados comparativos del tiempo de ejecución de las pruebas. En $P 1$, realizada mediante un teclado como periférico de entrada, se evidencia un menor tiempo de ejecución con respecto al tiempo empleado en $P 2$, donde se realiza la misma tarea de $P 1$ pero con el dispositivo BUCLE como periférico de entrada.

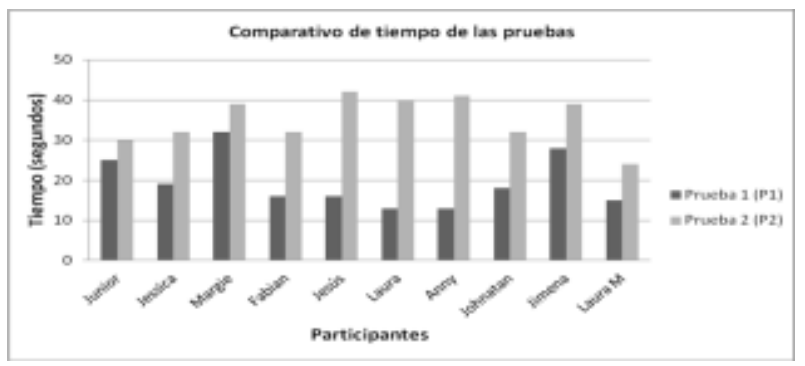

Figura 12. Comparativo de tiempo de las pruebas)

La respuesta motora mayor en un dispositivo como el teclado, se presenta por la cercanía de las 
teclas a presionar; también se observó que los participantes no usaban un único dedo para presionar las teclas y mantenían su mano lo más cerca posible a la zona donde estaba ubicada la tecla de interés, tal y como se acostumbra en los videojuegos. Lo anterior reduce el tiempo de la prueba. Es de anotar también que para los usuarios fue más "sencillo" el uso del teclado, en comparación con el dispositivo propuesto, ya que los movimientos necesarios para el uso de Bucle eran más complejos y lentos. Se observa una considerable diferencia en tiempos de realización de la tarea, de entre 10 y 25 segundos aproximadamente. Dicha diferencia se considera inadecuada en los videojuegos (Milán, Tornay, \& Quesada, 2005). Sin embargo, esto reafirma la aplicación del dispositivo propuesto en videojuegos donde el tiempo de respuesta pueda ser considerablemente alto, especialmente con usuarios en condición de desventaja motriz.

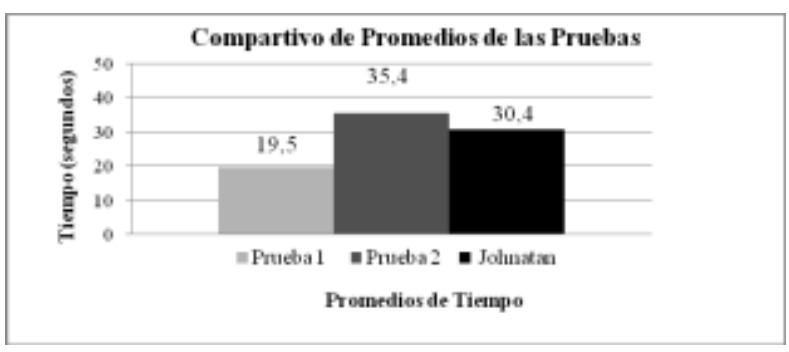

Figura 13. Comparación de tiempo promedio

En la figura 13 se observa un comparativo del tiempo promedio de las pruebas, contrastado con el tiempo de la prueba desarrollada por Johnatan. En esta figura se observa un tiempo promedio para $P 2$ de $35.4 \mathrm{~s}$; de igual manera el tiempo de Johnatan es de $30.4 \mathrm{~s}$, siendo menor al tiempo gastado por las personas en condiciones físicas normales. Se evidencia una diferencia a favor de Johnatan, reduciendo en términos de tiempo, la desventaja presente por la discapacidad física.

En la figura 14 se muestran los resultados de la eficacia obtenidos en P2. La eficacia promedio es de $57 \%$, y aunque se considera baja, se observa que la diferencia entre la eficacia obtenida por

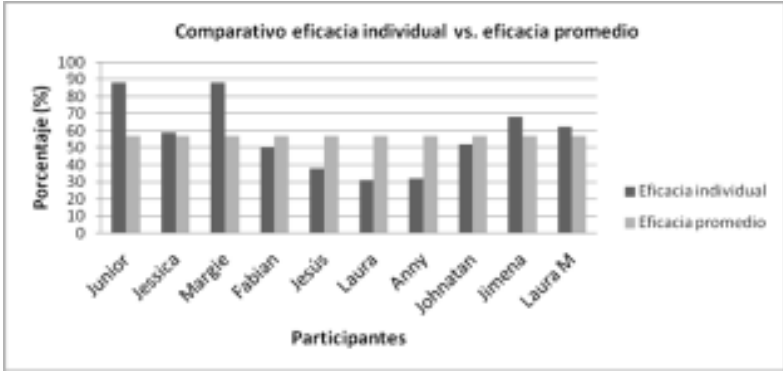

Figura 14. Comparativo de Eficacia Individual contra Eficacia Promedio.

Johnatan con $52 \%$ y la eficacia promedio de los demás participantes, es considerablemente baja, siendo de $5 \%$. Esto indica que la capacidad de desarrollar la tarea por parte de la persona con cuadriplejía, es muy cercana a la eficacia de una persona en condiciones físicas normales, cuando utilizan el dispositivo desarrollado.

En el desarrollo de pruebas se observaron algunas fallas en la precisión de los comandos, situación que fue corregida mediante una recalibración del sistema. Se observa la necesidad de realizar una calibración antes de iniciar su uso. Además, la precisión se puede mejorar con la utilización de giroscopios y acelerómetros con una sensibilidad superior.

\section{Conclusiones}

Se desarrolló BUCLE, un dispositivo electrónico de control como interface para un video juego, orientado a su uso en la interacción de personas en condición de cuadriplejía. En las pruebas realizadas se observó gran motivación del usuario en condición de discapacidad al poder desarrollar una actividad de entretenimiento, mediante un videojuego.

Se observó que el tiempo de la persona con cuadriplejía, al desarrollar la tarea, fue menor, en comparación con el tiempo promedio de las personas en condiciones físicas normales, con una diferencia de $5 \mathrm{~s}$. Esto muestra una reducción significativa de la brecha de rapidez generada por la condición de discapacidad motriz. 
La eficacia presentada por la persona en condición de discapacidad fue de $52 \%$ en comparación con el $57 \%$ obtenido por los participantes en condiciones normales, al momento de desarrollar la tarea usando BUCLE. La diferencia de 5\% muestra la capacidad de desarrollar la tarea de nuestro sujeto de estudio muy cercana a la capacidad de una persona sin condición de discapacidad.

Se propone el uso de BUCLE como un elemento en la terapia de orientación sicológica en la rehabilitación y tratamiento de pacientes con cuadriplejía, mediante la inclusión de videojuegos como mecanismo de integración social y familiar de los pacientes. Igualmente, se recomienda usar este dispositivo para el control de videojuegos donde el tiempo no sea un factor predominante, que afecte la buena experiencia de juego. Así mismo se sugiere su uso frecuente, con el fin de asimilar el aprendizaje mediante la práctica constante.

\section{Rerefencias}

Céspedes, G. M. (2005). La nueva cultura de la discapacidad y los modelos de rehabilitación. Bogotá, Colombia.

DANE. (2010).http://www.dane.gov.co

Discapnet. (2013). Discapnet. Recuperado el 15 de 08 de 2013, de salud.discapnet.es/Castellano/ Salud/Discapacidades/DiscapacidadesNeurologi cas/Lesion Medula Espinal/Paginas/cover lesion.aspx

Fanucci, L., Lacopetti, F., \& Roncella, R. (2011). A console interfaces for game accessibility to peopla with motor impairments. Publicado en Consumer Electronics - Berlin (ICCE-Berlin), 2011 IEEE International Conference. Alemania. ISBN 12273337.pp. 206-210
Grammenos, D., \& Savidis, A. (2009). Designing universally accessible games. Comput. Entertain. 7, 1, Article 8 (February 2009), 29 pages. DOI $=10.1145 / 1486508.1486516$

Huleves, Z., Aguayo, G., Lama, R. 2009. Diseño para la usabilidad de productos. Técnica industrial V. 282-2. p. 45-51. Fundación Técnica Industrial. Madrid, España

Learning Radiology. (2013). Recuperado el 31 de Agosto de 2013, de http://www.learnin gradiology.com/archives2008/COW\%20302Exten sion\%20Teardrop\%20Fx/hangmanbox.html

Maikel, V. S. (2012). Anatomía y exploración física de la columna cervical y torácica (2 ed., Vol. 2). Costa Rica: heredia.

Medical Exhibits. (2013). Recuperado el 31 de Agosto de 2013, de http://www.medical exhibits.com/medical_exhibits.php?exhibit $=0900$ 8_01XC\&query=spine\%20cervical\%20thoracic\%2 0C3\%20C4\%20C5\%20C6

Milán, E., Tornay, F., \& Quesada, J. (2005) La repetición de respuesta en situaciones de cambio de tarea. Departamento de Psicología Experimental y Fisiología de Conducta. Universidad de granada, España.

Nintendo. (2013). Nintendo.com. Recuperado el 10 de Septiembre de 2013, de http://www.nintendo. com/games/detail/B-99y_Rypyy 1 x0QOR OXfPD5OwUF9Hm_y

Plaza, N., \& Aperador, W. (2013) Technology in Locomotion and Domotic Control for Quadriplegic. Biomedical Engineering Conference (SBEC), 2013 29th Southern. ISBN 978-1-47990624-6 pp. 99-100. Miami, Florida. 
RadiologySpirit. (2013). Recuperado el 31 de Agosto de 2013, de http://3.bp.blogspot.com/_ FYzmMSSuvWc/TA4EP8wnrSI/AAAAAAAAEiQ/b0I MKIp9Dk4/s1600/obese-fig1.jpg

Rand D., N. G. (2012). Counting repetitions of upper extremity movements while playing videogames compared to traditional therapy: implications for stroke rehabilitation. 9th Intl Conf. Disability, Virtual Reality \& Associated Technologies, 487-490.

Readiology Assistant. (2013). Recuperado el 31 de Agosto de 2013, de http://www.radiology assistant.nl/en/p49021535146c5/spine-cervicalinjury.html

Spine-health. (2013). Spine Health. Recuperado el 15 de Agosto de 2013, de www.spine-healt.com

The eye writer. ( 2014). The eye writer. Recuperado el 18 de marzo de 2014, de http://eyewriter.org
Veckers, S. (2012). BBC mundo. Recuperado el 01 Diciembre de 2012, de http://redaccion.la mula.pe/2012/02/29/videojuego-se-maneja-conlos-ojos/lauraramirez/

Yankelevitz, K. (2010). Quad Control. Recuperado el 01 de Noviembre de 2012, de http://www.quad control.com

Zavala, J., \& Favela, J. (2012) Assessing muscle disease related to aging using ambient videogames. Conference on Pervasive Computing Technologies for Healthcare (PervasiveHealth), 2012 6th International. California USA. E-ISBN: 978 1-93-6968-42-5, Pp. 187-190.

Zhendou, H. (2000). PowerScan: a single-switch environmental control system for persons with disabilities. Bioengineering Conference, 2000. Proceedings of the IEEE 26th Annual Northeast. ISBN 0-7803-6341-8. pp. 171-172. 